\title{
Measuring and localizing individual bites using a sensor augmented plate during unrestricted eating for the aging population
}

\author{
Gert Mertes, Li Ding, Wei Chen, Hans Hallez, Jie Jia and Bart Vanrumste
}

\begin{abstract}
Food intake monitoring can play an important role in the prevention of malnutrition in the aging population, but traditional tools may not be adequate for use in this target group. These tools typically involve the use of questionnaires or food diaries that require manual data entry. Due to their time consuming nature, they are often incomplete, contain mistakes or not used at all. An alternative to self-reporting tools, in the form of a plate system that automatically measures the consumed food during the meal, is presented in this work. Furthermore, the system can estimate the location where each bite was taken on the plate. The system is compatible with an off-the-shelf plate that is mounted on top of a base station. Weight sensors are integrated in the base, allowing for easy removal and cleaning of the plate. Localization of bites is done by looking at the movement of the center of mass during eating. When used with a compartmentalized plate, the amount of consumed food per compartment can be measured. With prior knowledge of the type of food in each compartment, this can give an indication of calories and nutritional intake. We present a bite detection algorithm using a random forest decision tree classifier. Data from 24 aging adults (ages 52-95) eating a single meal with chopsticks was used to train and evaluate the model. Out of a total of 836 true annotated bites, the algorithm detected 602 with a precision and recall of 0.78 and 0.76 , respectively. By summing the weights of detected bites from each compartment, the algorithm was able to estimate the amount of food taken per compartment with an average error of $(8 \pm 8) \%$ of the portion size.
\end{abstract}

Index Terms-Food intake, bite detection, smart plate

\section{INTRODUCTION}

$\mathbf{M}$ ALNUTRITION is a frequent condition in the frailest of people in the aging population [1]. It is estimated that up to $15 \%$ of community dwelling and home-bound adults

This research was partially supported by the National Key R\&D Program of China (grant 2017YFE0112000), the Science and Technology Commission of Shanghai Municipality (grant 15441901601 and 16441905303), KU Leuven (grant IF/14/038) and Research Foundation Flanders (travel grant V410117N).

G. Mertes is with the Institute of Biomedical Engineering, Department of Engineering Science, University of Oxford, Oxford, U.K. and with the Oxford Suzhou Center for Advanced Research, Suzhou, Jiangsu, China. The work presented in this paper was done with the Department of Electrical Engineering (ESAT), KU Leuven, Leuven, Belgium. (e-mail: gertmertes@gmail.com)

L. Ding and J. Jia are with the Department of Rehabilitation Medicine, Huashan Hospital, Fudan University, Shanghai, China. (e-mail: leodin@163.com; shannonjj@126.com).

W. Chen is with the Center for Intelligent Medical Electronics, Department of Electronic Engineering, Fudan University, Shanghai, China (e-mail w_chen@fudan.edu.cn).

H. Hallez is with the Department of Computer Science, KU Leuven, Leuven, Belgium. (e-mail: hans.hallez@kuleuven.be).

B. Vanrumste is with the Department of Electrical Engineering (ESAT), KU Leuven, Leuven, Belgium. (e-mail: bart.vanrumste@kuleuven.be)

Corresponding authors: G. Mertes, J. Jia, W. Chen. aged over 65 are malnourished, while $45 \%$ are at risk [2], [3]. After institutionalization, prevalence of malnutrition greatly increases. Up to $60 \%$ of hospitalized elderly and up to $85 \%$ of nursing home residents show signs of undernourishment [4]. There is a direct link between health status and malnutrition in older adults. Malnutrition is associated with decreased muscle strength, poorly healing wounds, an increased hospital admission length and increased hospital mortality rate [5]. Furthermore, malnourished elderly are more prone to develop pressure ulcers and infections [6]. Preventing malnutrition by means of a targeted nutritional intervention could greatly improve the quality of life. Early recognition and treatment should therefore be included in the routine care of every elderly [2], [7]. Typical nutritional intake monitoring methods rely on manual data-entry, such as questionnaires and selfreporting food diaries [8]-[10]. While these can be an effective tool in nutritional screening to prevent malnutrition, they typically have to be completed with the help of a care professional. In the case of home-bound elderly adults receiving care at home, they are typically not used at all unless prescribed by a physician. Self-reporting methods such as dietary recall or food-diaries have similar shortcomings. Due to their time consuming nature, they may contain mistakes or data may be incomplete due to subjects not adhering to the monitoring protocol [8].

A possible method of objectively measuring food-intake without relying on self-reporting tools is through the use of sensors that automatically capture eating activity. There are several methods to accomplish this. Wearables can be used to detect movements of the body associated with eating activity. Chewing activity can be detected with sensors worn on the head or neck [11]-[15] while other body movements can be detected with wearables worn on the wrist or torso [16], [17]. These methods have the advantage of being passive and offer high performance, at the cost of comfort. Depending on the type of sensor, this method may be suited for use in the elderly [13], but lack the ability to measure quantity. Another approach is to use imaging techniques to detect and quantify types of food present on the plate [18]-[22]. The weight of the food can be estimated using 3D-reconstruction. The advantage of this approach is the low entry point if a smartphone is used to take pictures. The disadvantage is that manually taking pictures, often more than one per meal, is inconvenient. In the aging population, smart phone usage may also prove difficult [23]. Egocentric video solutions such as the one presented in [22] can be comfortable to use, but 3D- 
reconstruction can only provide an estimate of the consumed food. To accurately detect the amount of food consumed, weight sensors can be embedded in the kitchen or eating surface [24]-[27]. This, however, often requires an extensive adaptation to the existing infrastructure and measurements can only take place at the installation location. In previous work, we presented a standalone smart plate system fitted with weight sensors that can measure the weight and location of individual bites during meals [28]. The advantage of this system is that it can be used anywhere and is compatible with an off-the-shelf polymer plate that resembles those already used by nursing homes and home delivery food services. The hardware is built into a standalone base station and the location detection can be done without embedding sensors into the plate. This results in a low entry point and allows the plate to be used in uncontrolled environments such as nursing homes, hospitals or at home.

In this paper, we present a new algorithm capable of detecting individual bites during unrestricted eating for use with the smart plate system. The algorithm is based on a supervised Random Forest classifier. To evaluate the system in a real-world environment, the classifier is trained with data from 24 aging adults recorded in a hospital. Our bite detection algorithm is also compared to 3 related state-of-the-art algorithms [26], [29], [30]. Furthermore, the bite localization algorithm previously presented in [28] is combined with the new bite detector and evaluated on the dataset to test the feasibility of the approach during unrestricted eating.

\section{RELATED WORK}

Similar to the smart plate, Mattfeld et. al previously proposed the Universal Eating Monitor (UEM), a table embedded scale to measure individual bites during eating [26]. While the UEM is built into the table surface, the measuring methodology is similar: a weight sensor is used to measure food intake over time. An algorithm is proposed in [26] to measure individual bites using a rule based model. Regions of stability are first detected in the weight signal. Bites are consecutively detected by applying a set of threshold rules to transitions of stable regions.

Another device closely resembling the smart plate is the Mandometer. It is a standalone weight scale that is placed on the table and the plate with food is placed on top of it during eating. Similarly to the UEM, it can be used to continuously measure food intake during eating. Papapanagiotou et. al demonstrated three different algorithms to calculate the Cumulative Food Intake (CFI) curve from the raw data [29]. The CFI curve is a processed version of the raw weight signal that shows the amount of consumed food since the start of the meal. The authors present an algorithm named Greedy Quadratic Fitting (GQF). GQF uses a rule based model to detect food additions (FA). These are events where food is added to the plate. In order to eliminate the need for predefined thresholds in the rules, a low threshold is used as standard (which results in high recall, but low precision) and each candidate FA is evaluated based on the previous FA. This is done using the mean-squared error of two quadratic curves fitted to the FA (one rejecting it and one accepting it).
In more recent work, Papapanagiotou et. al presented another approach that models the meal with Context-Free Grammar (CFG) [30]. After pre-processing, the weight signal is segmented and a CFG terminal symbol is assigned to each segment (such as bite, food addition and artifact). A parse tree is constructed from the CFG string and used to calculate the CFI curve. From the CFI curve, individual bites can be measured.

In our previously published work on the smart plate [28], we presented the methodology of using a plate with multiple weight sensors to perform a localization of where food is taken from the plate. The localization algorithm, however, relies on an accurate detection of the bite in time. In [28], a rudimentary bite detector based on a simple moving-average and step detector is used. Furthermore, the algorithm was evaluated on data recorded in a lab environment with restrictions placed on the user. As we will show in Section III, real-world conditions differ greatly from restricted lab conditions. The bite detector presented in [28] would perform poorly in real-world conditions as the assumption is made that every decrease in weight after processing corresponds to a bite. A new approach is therefore presented. Furthermore, the bite localization algorithm was not evaluated on multiple measurements with varying food types. The purpose of this work is therefore to show the feasibility of the approach during realistic eating conditions on a larger dataset.

Restricted eating

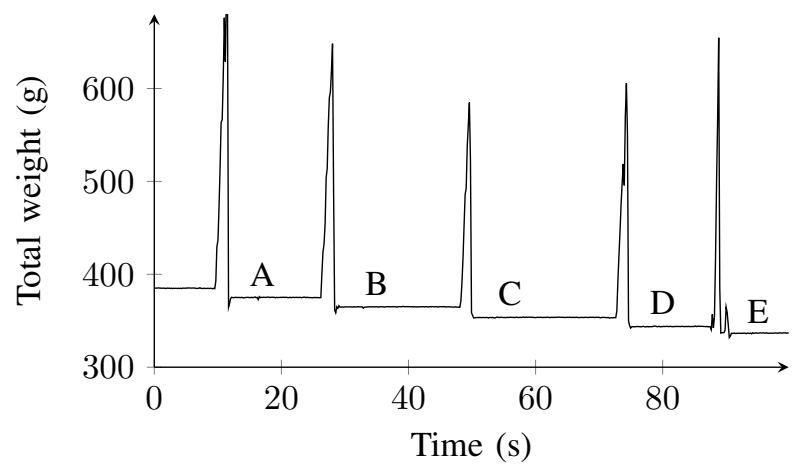

Unrestricted eating

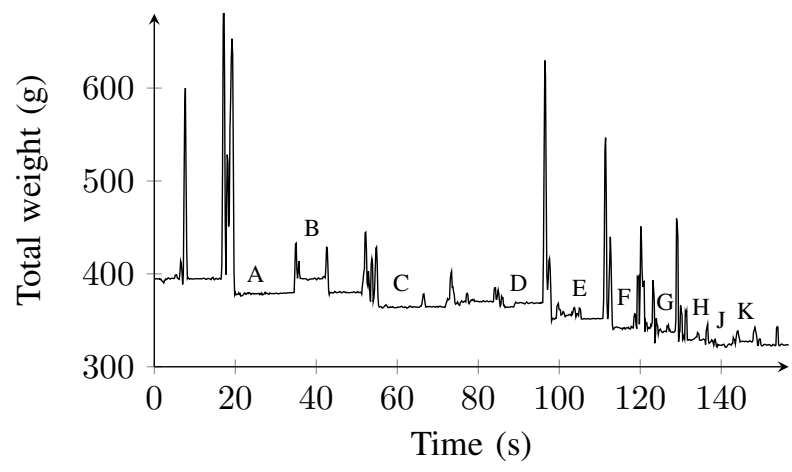

Fig. 1. Restricted eating (top) versus unrestricted eating (bottom) with the plate system. In the top figure, every decrease in weight corresponds to a bite. In the bottom figure, labels $\mathrm{C}$ and $\mathrm{D}$ are partial bites while $\mathrm{B}$ and $\mathrm{K}$ are periods where the utensils are rested on the plate. The remaining labels are single bites. 


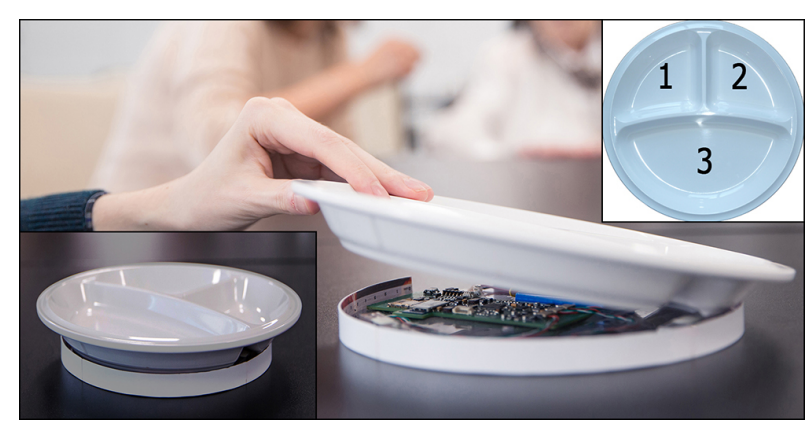

Fig. 2. The plate system: base station and plate. The polymer plate contains three compartments (numbered 1 to 3 ).

\section{RESTRICTED VS UNRESTRICTED EATING}

Restricted eating refers to the consumption of a meal, typically in a research or lab environment, where the subject is asked to change their regular eating behavior. The subject may be asked to eat slower, limit their interaction with the food, refrain from cutting or re-arranging food, etc. This is illustrated in Figure 1. The top shows a segment of plate data during restricted eating. All labels correspond to a single bite. In this case, food was served pre-cut and the subject was instructed to limit their interaction with the plate (e.g.: no cutting). A bite is typically characterized by a large spike coming from scooping, pushing or stabbing into the plate to pick up the bite, followed by a decrease in weight and a period of stability where the subject brings the bite to the mouth. In this signal, every decrease in weight can be considered a bite. The bottom shows an example of unrestricted eating. The signal appears a lot more noisy due to an increased amount of interaction with the plate and identifying bites is no longer straightforward. Bites are still characterized by the same pattern as before, but due to an increased eating speed and interaction with the plate, a decrease in weight no longer corresponds to a bite by default. Furthermore, the data may contain partial bites, where the subject returns a partially eaten piece of food back to the plate. In this example, labels $\mathrm{C}$ and $\mathrm{D}$ are partial bites, labels $\mathrm{B}$ and $\mathrm{K}$ are regions where utensils are temporarily placed on the plate and the remaining labels are single bites.

\section{Methodology}

\section{A. Plate system}

The plate system consists of an off-the-shelf polymer plate with three compartments that mounts onto a base station, as shown in Figure 2. The polymer plate is mounted using a reversible pressure fit connection that allows the plate to be easily removed for serving and cleaning. The base station contains three load-cells placed around the circumference in $120^{\circ}$ intervals. Data acquisition is done by a custom embedded system. The system is battery powered and data can be stored on an SD card or sent to an external device via Bluetooth. The three load-cells are sampled simultaneously at a selectable sample rate of $6 \mathrm{~Hz}, 12 \mathrm{~Hz}$ or $24 \mathrm{~Hz}$. The accuracy of the total measured weight is 1 gram.
TABLE I

DATASET PROPERTIES

\begin{tabular}{l|l}
\hline Participants & 24 \\
Average participant age & $63 \pm 10$ \\
Total meal duration & $198 \mathrm{~min}$ \\
Mean meal duration & $(11 \pm 5) \mathrm{min}$ \\
Mean meal weight & $(318 \pm 94) \mathrm{g}$ \\
Number of true bites & 836 \\
\hline
\end{tabular}

\section{B. Data}

Data was collected at Fudan University Huashan hospital Jing'An branch in Shanghai, China. The study was approved by the Huashan Hospital Institutional Review Board, approval reference number KY2013-163. Data was collected from 24 subjects: 12 male and 12 female. Table I shows the participant and dataset characteristics. All participants were healthy older adults that could independently eat. Each participant gave a written informed consent. Food was served on our plate system and each participant consumed 1 meal using chopsticks. Hospital food was served for the majority of subjects, while some preferred to bring food from an outside restaurant. No restrictions were placed on the type of food consumed, the only requirement was that it could be served in compartments on the plate. Before and after the meal, the total weight and weight of the food in each compartment was recorded with a kitchen scale. This was done to have a reference value of the total consumed food as well as the food consumed from each compartment so that this can be compared to the output of the compartment detection algorithm. A camera was used to record participants as they ate. Video was captured synchronously with the plate system and was later used to annotate the true bites. Labeling was done by the author. The time and location (compartment 1,2 or 3) of each bite is labeled. The bite time is the moment when a piece of food is observed to be lifted up from the plate. While there is some slack in the labeling, this is accounted for in the algorithm and explained in Section IV-C. Labels were generally placed within $1 \mathrm{~s}$ of the time when the weight decrease occurs.

\section{Bite detection}

The bite detection algorithm is centered around the idea that a bite is always preceded and followed by a stable region. The weight of a bite is measured by calculating the difference in weight from the stable regions surrounding the bite. Correctly identifying these regions is paramount in order to correctly measure and localize the bite. To determine a region of stability, a moving window with a length of $1 \mathrm{~s}$ is first passed over the total weight signal. The total weight signal is the sum of the three load cells after calibration. The total signal is used for bite detection since only this signal reliably decreases whenever a bite is taken. The weight on an individual sensor can actually increase after a bite, if the center of mass shifts towards that sensor. If the standard deviation of the data in the window is smaller than a threshold value, the window is considered to be stable. The threshold value was set at 3 gram. Both window length and threshold were chosen based on the findings in [26], since the approach is 


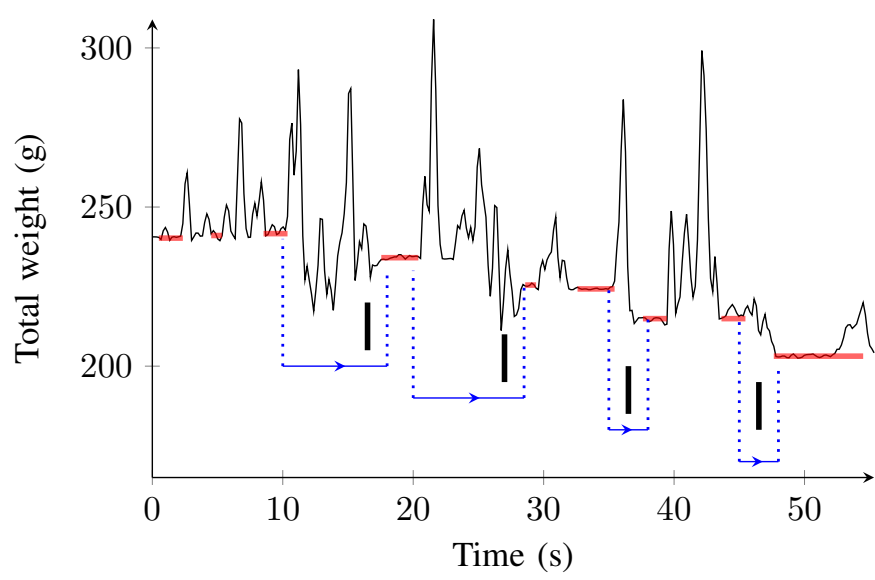

Fig. 3. Plot showing the stable regions in red (horizontal thick lines), extracted from the raw total weight signal. The thick vertical lines are the true annotations labeled by an observer. The dotted lines and blue arrows indicate the start and stop segments of each bite.

similar. These parameters have an impact on performance. Too large values will lower recall, while too small values will lower precision. After evaluation, we found that these values also produce the best results for our dataset. In a second step, consecutive windows of stability are merged together, forming a single region of stability. The values within each window are replaced with the mean value over the entire region. Figure 3 shows the result of this process on a segment of data obtained during unrestricted eating. The total weight signal is shown. The thick horizontal red lines are the detected regions of stability. The blue arrows indicate which pair of stable regions corresponds to a bite. These are deduced from the labeling done by an observer during the meal. The labels are shown with thick vertical lines. Note that annotation labels also contain the compartment number but this is not shown in Figure 3.

Bites are detected by looking at the relative position of regions of stability. Our work builds on the approach previously presented by Mattfeld et. al [26], but instead of manually creating the rules for each situation, a decision tree is trained to find the optimal combination of features and thresholds to detect a bite. A Random Forest (RF) classifier, from the MATLAB 2018a Machine Learning toolbox, is trained to distinguish between bites and no-bites. At this stage, both single and partial bites are grouped together under a single label. The TreeBagger function is used to train the ensemble. Number of trees is chosen at 50. Due to the nature of the classifier, which averages the classification results of all trees in the ensemble, a larger number of trees will generally result in better performance. During training and testing of the algorithm, we found that after 50 trees, the performance increase was negligible. TreeBagger uses fitctree to train the individual trees. Model parameters for both functions are left as default. The number of variables to sample at each decision split is the square root of the number of features, which is 4 in our case. Gini's Diversity Index is used as split criterion for construction of the individual trees. Trees are not pruned, since the RF algorithm already reduces overfitting [31].
Given a total number of stable regions $M$, the weight and time information of each stable region is stored in a vector $\boldsymbol{S}_{\boldsymbol{i}}=\left[W_{i}, T 1_{i}, T 2_{i}\right]$, with $W_{i}$ the mean weight value of the region and $T 1_{i}$ and $T 2_{i}$ the respective start and stop times of the region, for $i=1, \ldots, M$. A feature vector is then constructed that combines information from 3 consecutive stable regions, defined by:

$\boldsymbol{X}_{i}=\left[\left(W_{i}-W_{i+1}\right),\left(W_{i}-W_{i+2}\right),\left(T 2_{i}-T 1_{i+1}\right),\left(T 2_{i}-T 1_{i+2}\right)\right]$

for $i=1, \ldots, M$. It combines the differences in weight between the first and second and between the first and third consecutive stable region. This is done for all stable regions in the signal. Three regions are combined per feature vector to give the model the ability to look ahead and increase the robustness against situations where food is temporarily removed but placed back that may otherwise be misclassified as a single bite. The complete feature matrix is written as $\boldsymbol{X}=\left[\boldsymbol{X}_{1}, \boldsymbol{X}_{2}, \ldots, \boldsymbol{X}_{\boldsymbol{M}}\right]^{\mathrm{T}}$.

Labeling for training is done based on the true bite labels assigned during the video annotation. True bite labels were always placed at the time immediately after a bite was removed from the plate. If a single true bite label is located between $T 2_{i}$ and $T 2_{i+1}$, the instance $\boldsymbol{X}_{\boldsymbol{i}}$ is labeled a bite. The stop time, instead of the start time, of the second stable region is used to allow for some inaccuracy in the labeling. If no true label is found, the instance is labeled a nobite. A special situation occurs if multiple true labels are found between $T 2_{i}$ and $T 2_{i+1}$. In this case, the instance is not unequivocally assignable to either class. This case can be called a missbite, because the algorithm is unable to detect these bites due to there only being one instance associated with these true bites. The primary goal of the classifier is to detect individual bites so that they can be measured, localized and assigned to a compartment in order to detect the total consumed weights per compartment. In the event of a miss-bite, the individual true bites are no longer detectable and cannot be localized on the plate. It is thus reasonable to assign a miss-bite to the negative class of the classifier and assign it a nobite label. Doing so, however, has the potential to introduce noise in the classifier. A miss-bite corresponds to a decrease in weight, similar to a single bite. This can bias the classifier to miss single-bites with a similar decrease in weight.

To evaluate the impact of miss-bites, two test cases are evaluated. In test case 1 , miss-bite instances are assigned the nobite label during training, in an attempt to train a classifier that only detects single bite events. In test case 2, miss-bites instances are assigned the bite label. This alternative classifier detects both normal and miss-bite events, effectively creating a classifier that detects epochs of eating behavior in general.

\section{Bite Localization}

Using more than one load-cell adds the ability to localize bites as they are taken from the plate. This is accomplished by looking at the movement of the plates' center of mass. When a bite is taken from the plate at a certain position, the center 


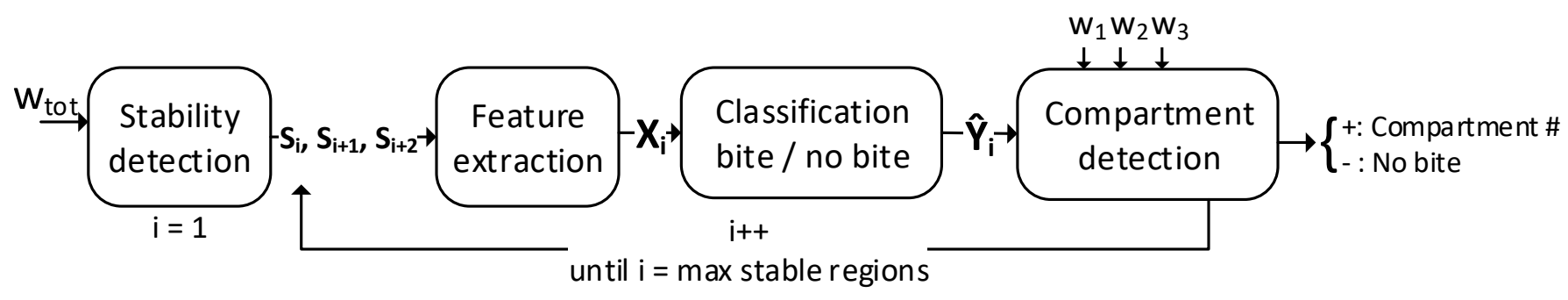

Fig. 4. The complete detection pipeline.

of mass will shift in the opposite direction with a distance proportional to the bite weight. By calculating the center of mass before and after the bite, the bite can be localized. This results in equations (1) and (2) [28], with $x$ and $y$ the bites' location in the horizontal plane, $\Delta F_{i}$ the change in force on sensor $i$ from before to after the bite, $\Delta F_{\text {tot }}$ the total change in force on all sensors and $x_{i}$ and $y_{i}$ the distance from the origin to sensor $i$.

$$
\begin{aligned}
& x=\frac{1}{\Delta F_{\text {tot }}} \sum_{i=1}^{3} \Delta F_{i} x_{i} \\
& y=\frac{1}{\Delta F_{\text {tot }}} \sum_{i=1}^{3} \Delta F_{i} y_{i}
\end{aligned}
$$

The values $\Delta F_{\text {tot }}$ and $\Delta F_{i}$ are calculated directly from the output of the bite detection algorithm. Since the bite detection algorithm works under the assumption that ever bite is directly surrounded by a stable region, $\Delta F_{\text {tot }}$ and $\Delta F_{i}$ can be found by taking the weight difference between consecutive stable regions that were detected as a bite.

\section{E. Complete algorithm}

Figure 4 shows the complete algorithm pipeline. The stability detection block takes the total weight signal $w_{t o t}$ and looks for stable regions $S$. The stable region $S_{i}$ and its two subsequent neighbors, $S_{i+1}$ and $S_{i+2}$, are passed to the feature detection block. The feature extraction block outputs the vector $\boldsymbol{X}_{\boldsymbol{i}}$. The feature vector is then classified by the bite detection model. The prediction $\hat{Y}_{i}$ is passed to the compartment detection. The compartment detection attaches a compartment number to each positive detection. Negative detections are passed through with no change, because the detector determined there is no bite to localize. The compartment detection takes the total weight of the bite as well as the weights measured by each individual load cell and localizes the bite using formulas (1) and (2). Total weight of the bite is calculated as $W_{i}-W_{i+1}$ for single bites and $W_{i}-W_{i+2}$ for partial bites. Bite type is determined using a simple evaluation: the instance is a single bite if $\left(W_{i}>W_{i+1}>W_{i+2}\right)$ and a partial bite if $\left(W_{i}>W_{i+1}<W_{i+2}\right)$. The coordinates are then compared to the layout of the polymer plate and a compartment is assigned to each detection. The process is repeated for each stable region. At the output of the algorithm, each instance will have been assigned a bite weight and a compartment number or a nobite label.

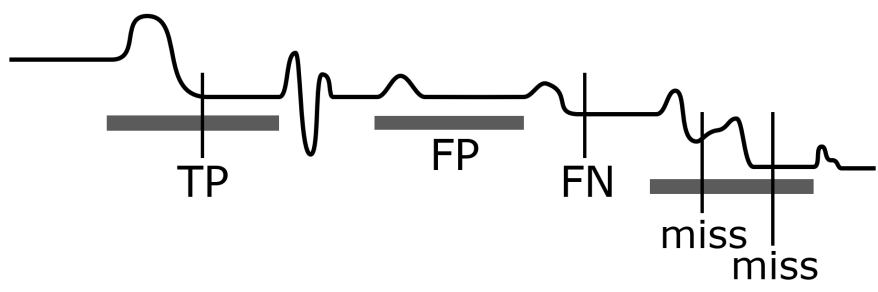

Fig. 5. The proposed evaluation scheme illustrated on a hand-drawn weight signal. Vertical lines are ground truth labels, thick horizontal bars are the detection range.

\section{F. Performance evaluation}

The classification model is evaluated using a leave-oneperson-out cross-validation scheme. Data from one person is used to test the algorithm and data from all other persons is used to train the classification model. This is done for each person in the dataset. Note that only the bite/nobite classifier is retrained in each iteration, the compartment detection is a deterministic model that remains unchanged. This scheme is chosen due to the limited size of our dataset. Rather than using a more traditional training/validation/test split, which would result in a small test set that may not be representative of the population, the leave-one-person-out scheme allows for each person in the dataset to be used for testing. This gives a better idea of the generalization of the model.

To evaluate the algorithm's performance, the output labels are compared to the ground truth labels obtained during annotation. Evaluation is done in a similar fashion as the labeling of the training data. The predicted bites contain the beginning and end times of a bite, their weight and their predicted location. These are then compared to the annotated ground truth labels. The evaluation scheme is illustrated in Figure 5. If one true label occurs inside the start and stop time of a predicted bite, this is considered a true positive (TP). If no true label is found inside the detection range, the bite is considered a false positive (FP). If a true label is found in a region marked as negative (nobite), this is a false negative (FN). If no true label is found in this region, it is a true negative (TN). If multiple true labels occur within one predicted bite, this is a miss-bite and its evaluation depends on the test case. Assume a number of $k$ ground truth labels in a single missbite detection. In test case 1, where the classifier was trained to detect only single-bite events and miss-bites were assigned the negative class during training, this is considered $1 \mathrm{FP}$ and $k$ FN. The rationale is that since this type of instance was labeled a nobite during training, a detection should not have 
TABLE II

Bite Classification RESUlts. TC $=$ Test CASE. *MeAN total MEAL WEIGHT ERROR.

\begin{tabular}{|c|c|c|c|c|c|}
\hline & Papapanagiotou et. al [29] & Papapanagiotou et. al [30] & Mattfeld et al. [26] & Our approach (TC1) & Our approach (TC2) \\
\hline Precision & 0.58 & 0.72 & 0.79 & 0.74 & 0.78 \\
\hline Recall & 0.55 & 0.51 & 0.54 & 0.65 & 0.76 \\
\hline F1-score & 0.56 & 0.60 & 0.64 & 0.69 & 0.77 \\
\hline Weight error* $[\mathrm{g}]$ & $279 \pm 274$ & $34 \pm 22$ & $58 \pm 64$ & $45 \pm 83$ & $\mathbf{2 9} \pm 39$ \\
\hline \multicolumn{6}{|c|}{ Performance estimates } \\
\hline Precision & $0.56^{\mathrm{a}}$ & $0.71^{\mathrm{a}}$ & $0.81^{\mathrm{b}}$ & $0.76^{\mathrm{b}}$ & $0.77^{\mathrm{b}}$ \\
\hline Recall & $0.53^{\mathrm{a}}$ & $0.56^{\mathrm{a}}$ & $0.55^{\mathrm{b}}$ & $0.64^{\mathrm{b}}$ & $0.77^{\mathrm{b}}$ \\
\hline
\end{tabular}

a: Evaluated using our evaluation scheme of test case 2, by converting the timestamps into time periods.

b: Evaluated using the evaluation scheme of [30], by converting the time periods into timestamps.

occurred and is therefore a FP. Furthermore, the algorithm is inherently unable to measure each individual true bite and they are therefore assigned a FN. In test case 2, where missbite instances were assigned the positive class during training, a more relaxed approach is taken. Here, the prediction is considered $1 \mathrm{TP}$ and $k-1 \mathrm{FN}$. The rationale here is that one ground truth label can be matched to the detection, while the remainder were missed.

Furthermore, the positive detections from each leave-oneperson-out test are used to estimate the weight of consumed food from each compartment. The consumed food per compartment is obtained by taking the sum of the weights of bites detected in each compartment. This value is then compared to the true weight of consumed food and the error per compartment is calculated. Note that the true weight of consumed food is known because the food in each compartment was measured with a kitchen scale before and after each meal.

\section{G. Performance comparison}

The performance of our algorithm is compared with that of the following three algorithms: Papapanagiotou et. al (GQF) [29], Papapanagiotou et. al [30] and our implementation of the UEM algorithm presented by Mattfeld et al. [26]. All three are deterministic models that do not require training, they are therefore directly applied to each person in our dataset and the results are averaged. For the algorithm in [26], we implemented the single bite and partial bite. Drink events were omitted as our dataset does not contain this data. For the selection of threshold parameters $W_{1}, W_{2}$ and $W_{3}$, a similar approach to the one used in [26] was used. A random set of 5 meals was selected to look at the distribution of bite weights. The maximum single bite size in this subset was $21 \mathrm{~g}$. We therefore chose $W_{1}=21 \mathrm{~g}$. Similarly, partial bites were within the range of $40 \mathrm{~g}-160 \mathrm{~g}$. Based on this, we selected $W_{2}=40 \mathrm{~g}$ and $W_{3}=160 \mathrm{~g}$. Equation (2) of the single bite stage was also omitted, since we found that this only had a negative impact on performance. The algorithm is evaluated using the same evaluation scheme used in our test case 2 .

The author of [29] and [30] provided us with the bite detection results using their original algorithm. The same maximum bite threshold values of $20 \mathrm{~g}$ are used for both algorithms since they are almost identical to our chosen value of $W_{1}$. The evaluation methodology presented in Section $\mathrm{V}-\mathrm{B}$ of [30] is used to calculate the performance for both algorithms. Each detection is matched to at most one ground truth label and vice versa. The same boundary of $10 \mathrm{~s}$ is used to check for a ground truth label around each detected bite to allow for some delay in the labeling. Due to the nature of both algorithms, it is technically impossible to use the exact same evaluation scheme as the one we propose. Our algorithm produces time periods during which a bite has been detected, while algorithms [29] and [30] produce a single timestamp when a bite is detected. This makes it impossible to have exactly the same evaluation schemes. Our definition of true and false detections is based on the presence of a true label in the detection time range, while the scheme in [30] uses the presence of a true label in a boundary of $10 \mathrm{~s}$ around the detection to determine if the detection is true or false.

To investigate the difference between both evaluation schemes, additional performance estimates can be calculated in order to achieve uniformity among the results. The detection time periods produced by our algorithm and Mattfeld et al. [26] can be converted into estimate timestamps by taking the mid point of the detection range, defined as $\frac{t_{\text {start }}+t_{\text {stop }}}{2}$. The estimated timestamps can then be evaluated using the evaluation scheme of [30] to allow a more direct comparison. Similarly, the timestamps produced by [29] and [30] can be converted into estimate bite periods by inflating them with half the average bite period duration - as produced by our algorithm - on each side. The average bite period produced by our algorithm is $8 \mathrm{~s}$. The estimated periods can then be evaluated using our proposed evaluation scheme.

\section{Results}

Table II shows the bite classification results of our approach and the three other algorithms. Precision and recall are calculated from the true and false detections. Also shown is the mean total weight error. The total meal weight is obtained by summing the weights of all detected bites. The mean total weight error is then calculated as the mean absolute deviation from the ground truth meal weights. The approach by Mattfeld et al. obtains the highest precision, while our approach is able to get the highest recall and lowest mean total weight error. This is encouraging, as it indicates that our approach is able to detect more bites in our dataset. Furthermore, our approach also has the highest F1-score, which is defined as $2 \times \frac{\text { precision } \times \text { recall }}{\text { precision }+ \text { recall }}$. The total meal weight error of Papapanagiotou et al. [30] is the second lowest and has less uncertainty 
TABLE III

CONFUSION MATRIX: BITE DETECTION (OUR APPROACH)

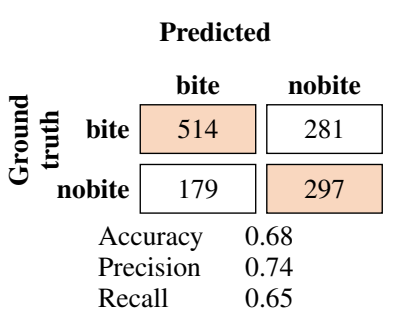

(a) Case 1

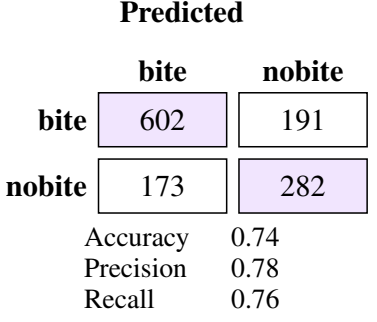

(b) Case 2

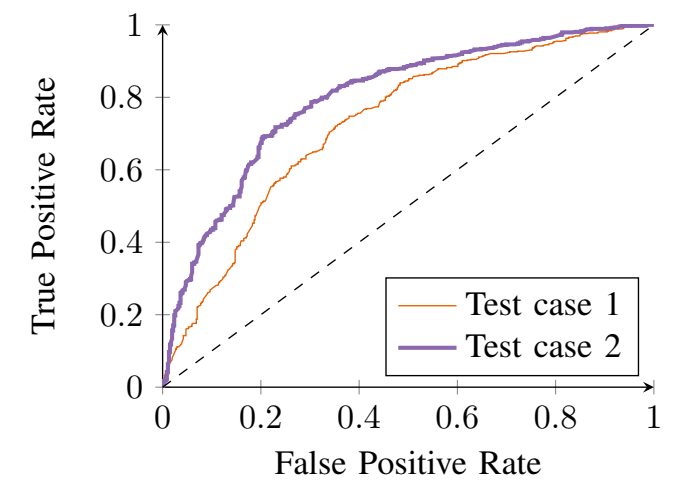

Fig. 6. ROC curves of the bite detection classifier for case 1 (thin orange) and case 2 (thick purple). $A U C_{\text {case } 1}=0.73, A U C_{\text {case } 2}=0.80$

than our approach. While [30] has a significantly lower recall, precision is similar. This indicates that their algorithm tends to aggregate bites taken in quick succession. The cumulative weight of these bites is still correctly measured, but the individual bite detections are missed.

The bottom part of Table II contains the performance estimates, as introduced in Section IV-G. Evaluating our approach and [26] with the evaluation scheme of [30] and vice versa does not have a significant impact on performance. While both schemes are slightly different due to the nature of the algorithms, the performance estimates show that the schemes are sufficiently similar to warrant comparison of the results.

Table III shows a confusion matrix for both test cases in our approach to illustrate the distribution of true and false detections. The outputs of each iteration of the leave-oneperson-out validation are summed together and shown here. The detection accuracy, recall and precision are indicated below the confusion matrix for each test case. Test case 2 performs the best with a precision and recall of 0.78 and 0.76 , respectively. In test case 1 , the classifier detected 38 miss-bites. These miss bites contribute $38 \mathrm{FP}$ and $83 \mathrm{FN}$ to the results. In test case 2, 43 miss-bites are included in the TP and a corresponding $58 \mathrm{FN}$. This shows that, with the exception of 5 instances, the classifier is not able to classify miss-bites separately from single-bites, as they are detected as a bite in both test cases. The marginal decrease in detected miss-bites in test case 1 does not outweigh the increase in performance in test case 2, particularly in the number of FN events. This supports our hypothesis that miss-bites events are not distinguishable from single-bite events and assigning them the nobite label during training has a negative impact on classifier performance. While test case 2 uses a more relaxed evaluation approach, the contribution of miss-bites to the total results is small and this test case is preferable.

Figure 6 shows the ROC curve for the bite classifier for both test cases $\left(A U C_{\text {case } 1}=0.73, A U C_{\text {case } 2}=0.80\right)$. The scores used to construct the curve are the ratios of number of bites to nobites for each leaf in the tree ensemble.

Table IV shows the confusion matrix when taking into account the predicted compartment numbers for each bite. This is an extended version of Table III: the positive detections under the bite label are now split up according to their predicted and real compartment. The FP and FN are still shown under the nobite label, but are also split up based on their predicted and real compartment respectively. This is the result of FPs that were wrongly classified as being a bite, and thus were assigned a wrong compartment because there was no bite in the first place, or FNs that should have been assigned a compartment, but were missed because the compartment detection only attempts to localize the positive detections of the classifier. The numbers between brackets are the amount of additional miss-bite detections that contain true bites from the same compartment. In this situation, the compartment of the true bites is assigned as real compartment of the miss-bite. There are 26 miss-bites, not included in the confusion matrix, where a real compartment could not be assigned because the true bites came from different compartments.

Figure 7 shows a box plot of the weight error per compartment over all measurements. The error for each measurement is normalized to the total weight of each meal. A negative error means that the algorithm under-reported the actual food intake per compartment. A positive error is the result of overreporting. The results are more biased towards a negative error, which can be explained by the fact that the bite detection reported more FN than FP. Similar to the results of the bite detection algorithm, test case 2 performs the best with lower maximum errors, lower median error and a lower interquartile range (IQR). The average error and std. dev. per compartment for test case 2 is $(12 \pm 12) \%,(5 \pm 5) \%$ and $(8 \pm 9) \%$ for compartment 1, 2 and 3 respectively. This is the error relative to the total portion size.

\section{DISCUSSION}

The biggest challenge during unrestricted eating is dealing with a variety of different situations that can arise when a subject interacts with the plate and food. Without additional context information, it can be difficult, or sometimes impossible, to extract individual bites from the weight signal. One factor contributing to misclassifications are bites taken in quick succession without allowing the plate to stabilize and the re-arranging of food. Almost all of the measurements in our dataset contained segments of data with multiple bites, but with no distinguishable stable regions in-between, making the identification of single bites impossible. In this situation, however, the cumulative size of the bites could often still be determined. Another source of errors is the misclassification 
TABLE IV

CONFUSION MATRIX: BITE LOCALIZATION (OUR APPROACH)

\section{Predicted compartment}

\begin{tabular}{|c|c|c|c|c|}
\hline \multirow{4}{*}{ 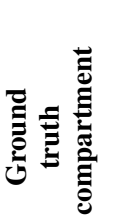 } & 1 & 2 & 3 & nobite \\
\hline & 60 & 19 & 16 & 58 \\
\hline & 9 & 96 & 14 & 80 \\
\hline & 17 & 42 & 241 & 143 \\
\hline nobit & 26 & 63 & 90 & 297 \\
\hline
\end{tabular}

(a) Case 1

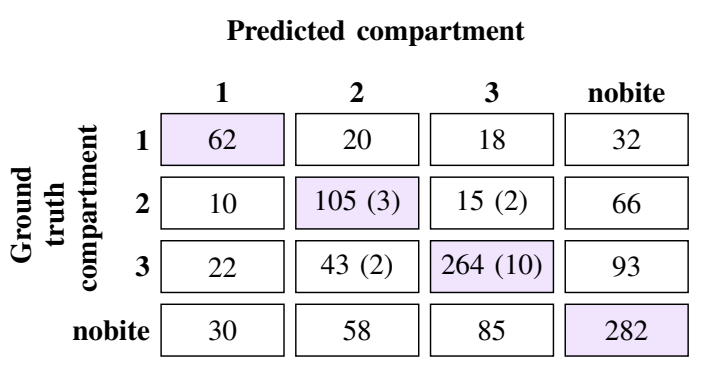

(b) Case 2

Note 1: because the localization algorithm can only localize the positive outputs of the classifier, it will also attempt to localize false positive bites and miss false negatives. These are shown under the nobite label. True negative bites are shown on the crossing of the nobite label.

Note 2: the numbers between brackets indicate the amount of additional miss-bite detections that contain true bites all from the same compartment. In this case, the compartment of the true bites is assigned as the real compartment of the miss-bite. There are 26 remaining miss-bites where the true bites inside the miss-bite event come from different compartments. The latter are not included in the confusion matrix because no ground truth compartment can be unequivocally assigned.

of partial bites as single bites. If the weight signal is not stable while a partial bite is being taken, for example because the subject is using their off-hand to touch or lean on the plate, the replacement of the partial bite on the plate may be missed and the initial decrease in weight may be seen as a single bite. Some subjects had the habit of touching or leaning on the plate with their free hand while taking a bite, again preventing the plate from stabilizing. Furthermore. our dataset also contains some behavioral patterns that can be attributed to the use of chopsticks. A frequent occurrence in the data is the re-arranging of food in order to pick up the bite. Subjects would often poke at or shove pieces of food to align them in an orientation that made them easier to pick up with chopsticks. In some cases, a single bite of food would be picked up multiple times from the plate and put back in order to get a better grip on it, before being consumed. This in turn is a source of some false positives.

The use of chopsticks is also a limiting factor in this work. Since all test subjects used chopsticks, the algorithm was not evaluated with different utensils (spoon, knife, fork). It should also be noted that the algorithms presented in [29] and [30] - to which we compare our approach - were originally not designed nor evaluated for the use of chopsticks and

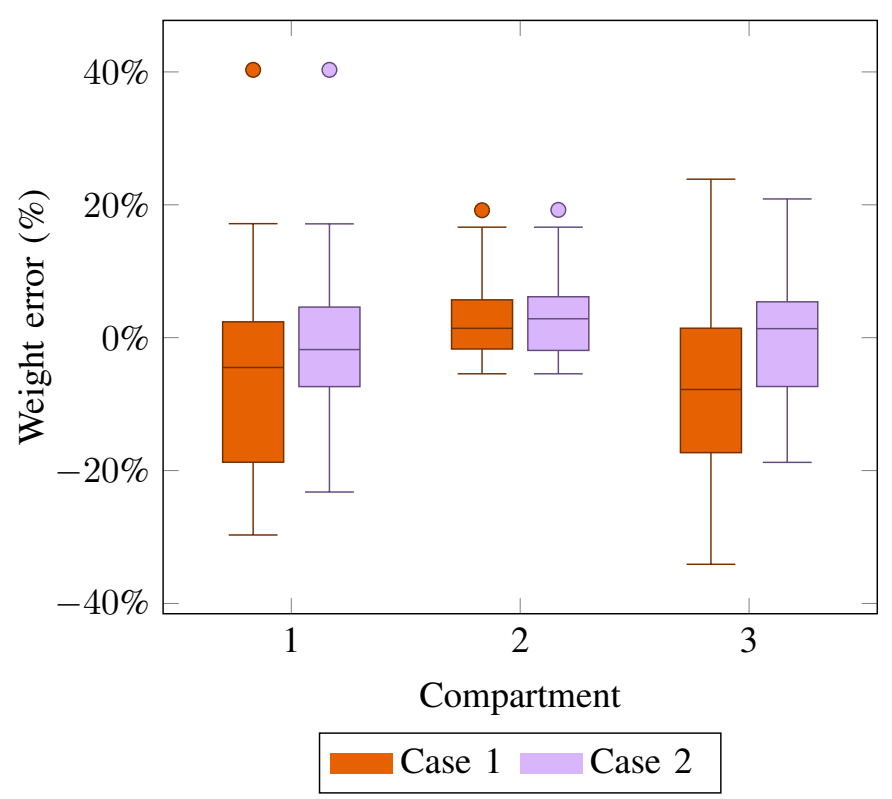

Fig. 7. Box plot showing the distribution of the weight error for our approach per compartment, relative to the total weight of the meal.

lack this data completely. The model presented in [26] was originally evaluated on a dataset containing 356 bites taken with chopsticks, but this only represented $1.5 \%$ of the total bites. Different utensils can have different footprints, which can impact different stages of the algorithm. Algorithms [26] and [30] do generate satisfactory results on our data, which shows the validity of their approach, but performance may be negatively influenced by our limited dataset. For a more generalized view, the proposed algorithm should be evaluated on a larger dataset with different types of utensils.

As shown in Table IV, true bites are biased towards compartment 3, containing 1.4 times more bites than compartments 1 and 2 combined. Compartment three is also the one with the most false negatives and false positives. This is because compartment 3 was used to serve rice in all but two measurements. The portion size of the rice served in compartment 3 - which is also the biggest compartment - was typically twice that of the other two compartments, resulting in more bites from this compartment. Rice was also one of the foods that would typically require more than one action to pick up from the plate, and thus introduce misclassifications.

Naturally, misclassifications will also result in an incorrect weight measurement. While the median weight error per compartment is situated around 0 and the boundaries of the 1.5 IQR interval are less than $10 \%$ of the total portion size, as shown in Figure 7, outliers of up to $40 \%$ are observed. The tight 1.5 IQR bands give an indication that the algorithm performs well for the majority of test data, while the large minima and maxima and few outliers indicate that there are still measurements where the algorithm performs poorly. All outliers observed in the box plot are from a single measurement where the test subject was touching or leaning on the plate for long periods of time. Similarly, the large maxima for compartments 1 and 3 are the result of another subject leaning on the plate for over half the duration of the meal. 
Even if the detected weight per compartment is incorrect, the total meal size may still be correctly measured. A bite may be classified in the wrong compartment, but its weight can still be measured correctly and added to the estimated total portion size. In some cases bites that were taken in quick succession were not individually detected, but their cumulative weight decrease was detected as one large bite and the cumulative weight correctly measured. On average, the algorithm was able to estimate the total portion size with an error of $29 \mathrm{~g}$. If we disregard the two measurements where test subjects leaned on the plate for large periods of time, an activity that makes it almost impossible for the bite detection algorithm to perform well, the average error of the total portion size drops to $18 \mathrm{~g}$. One study found that nursing staff, on average, overestimate the amount of food consumed in nursing home residents by $22 \%$ [32]. For our dataset, with an average meal size of $318 \mathrm{~g}$, this corresponds to an error of $70 \mathrm{~g}$. Our algorithm is able to improve on that estimate. The automated plate system could further help reduce work load and provide more accurate intake estimation for nursing home residents.

The ability of the plate to measure the consumed food per compartment has the ability to lower the recording burden and increase adherence. In situations where extensive intake monitoring is required, the user no longer has to manually measure each individually consumed food. The weights of each compartment can be assigned to a food type to automatically measure calorie intake. This will still require either a manual step or a post-processing step (e.g.: using video imaging techniques to detect the food type), but it is already an improvement over traditional pen-and-paper techniques.

In some cases, the algorithm performed exceptionally well. In 9 measurements, the algorithm measured the weight of the food consumed in each compartment with an error of less than $15 \mathrm{gram}$. In one case, the meal consisted of dumplings that were served only in compartment 3 . In this measurement, the algorithm approached perfect accuracy, with no bites detected in compartment 1 and 2, $96 \%$ of bites detected in compartment 3 and with the total weight correctly estimated within $9 \mathrm{~g}$.

\section{CONCLUSION}

This work presents a standalone plate system that is able to measure the weight and location of individual bites during unrestricted eating. A supervised learning method is used to detect individual bites. We compared performance to three state-of-the-art algorithms and our approach was able to achieve the highest recall and lowest mean total weight error, while achieving second best precision. The novelty of our approach is the ability to measure bite location without embedding sensors in the plate. An off-the-shelf plate with compartments is mounted on top of three load cells and the weight distribution of each sensor in time is used to localize bites on the plate. The weight of bites taken from each compartment can be estimated. With prior knowledge of the food present in each compartment, the amount of calories can be automatically estimated. The proposed system can provide an added value in situations where self-monitoring is required. The system is mobile and requires minimal user interaction, making it suitable for use by the aging population.

\section{ACKNOWLEDGMENT}

The authors thank Vasileios Papapanagiotou for applying the algorithms in [29] and [30] to our dataset and providing us with the results.

\section{REFERENCES}

[1] R. DiMaria-Ghalili and E. Amella, "Nutrition in older adults: Intervention and assessment can help curb the growing threat of malnutrition." American Journal of Nursing, vol. 105, pp. 40-50, 2005.

[2] L. Donini, P. Scardella, L. Piombo, B. Neri, R. Asprino, A. Proietti, S. Carcaterra, E. Cava, S. Cataldi, D. Cucinotta, G. Di Bella, M. Barbagallo, and A. Morrone, "Malnutrition in elderly: Social and economic determintants," The Journal of Nutrition, Health \& Aging, vol. 17, pp. 9-15, 2013.

[3] Nutricia, "Results of the NutriAction II study," 2013.

[4] L. Donini, C. Savina, M. Piredda, D. Cucinotta, A. Fiorito, E. Inelmen, G. Sergi, L. Dominguez, M. Barbagallo, and C. Cannella, "Senile anorexia in acute-ward and rehabilitation settings," The Journal of Nutrition Health and Aging, vol. 12, no. 8, pp. 511-517, 2008.

[5] E. Cereda, C. Pedrolli, A. Zagami, A. Vanotti, S. Piffer, A. Opizzi, M. Rondanelli, and R. Caccialanza, "Nutritional screening and mortality in newly institutionalised elderly: a comparison between the geriatric nutritional risk index and the mini nutritional assessment," Clinical Nutrition, vol. 30, no. 6, pp. 793-798, 2011.

[6] D. Volkert, L. Pauly, P. Stehle, and C. C. Sieber, "Prevalence of malnutrition in orally and tube-fed elderly nursing home residents in germany and its relation to health complaints and dietary intake," Gastroenterology research and practice, 2011.

[7] H. Lochs, C. Pichard, and S. Allison, "Evidence supports nutritional support," Clinical Nutrition, vol. 25, no. 2, pp. 177-179, 2006.

[8] L. Burke, M. Warziski, T. Starrett, J. Choo, E. Music, S. Sereika, S. Stark, and M. Sevick, "Self-monitoring dietary intake: Current and future practices," Journal of Renal Nutrition, pp. 281-290, 2005.

[9] O. Bouillanne, G. Morineau, C. Dupont, I. Coulombel, J.-P. Vincent, I. Nicolis, S. Benazeth, L. Cynober, and C. Aussel, "Geriatric nutritional risk index: a new index for evaluating at-risk elderly medical patients,' The American journal of clinical nutrition, vol. 82, pp. 777-783, 2005.

[10] E. Cereda, "Mini nutritional assessment," Current Opinion in Clinical Nutrition \& Metabolic Care, vol. 15, pp. 29-41, 2012.

[11] S. Passler and W.-J. Fischer, "Food intake monitoring: Automated chew event detection in chewing sounds," IEEE journal of biomedical and health informatics, vol. 18, no. 1, pp. 278-289, 2014.

[12] O. Amft, "A wearable earpad sensor for chewing monitoring," in Sensors, 2010 IEEE. IEEE, 2010, pp. 222-227.

[13] G. Mertes, H. Hallez, B. Vanrumste, and T. Croonenborghs, "Detection of chewing motion in the elderly using a glasses mounted accelerometer in a real-life environment," in Engineering in Medicine and Biology Society (EMBC), 2017 39th Annual International Conference of the IEEE. IEEE, 2017, pp. 4521-4524.

[14] R. Zhang and O. Amft, "Monitoring chewing and eating in freeliving using smart eyeglasses," IEEE journal of biomedical and health informatics, vol. 22, no. 1, pp. 23-32, 2018.

[15] E. Sazonov and J. Fontana, "A sensor system for automatic detection of food intake through non-invasive monitoring of chewing," IEEE Journal of Sensors, vol. 12, pp. 1340-1348, 2012.

[16] J. Fontana, M. Farooq, and E. Sazonov, "Automatic ingestion monitor: A novel wearable device for monitoring of ingestive behavior," IEEE Transactions on Biomedical Engineering, pp. 1772-1779, 2014.

[17] O. Amft and G. Tröster, "Recognition of dietary activity events using on-body sensors," Artificial Intelligence in Medicine, vol. 42, 2008.

[18] H. He, F. Kong, and J. Tan, "Dietcam: Multiview food recognition using a multikernel svm," IEEE journal of biomedical and health informatics, vol. 20, no. 3, pp. 848-855, 2016.

[19] R. Weiss, P. J. Stumbo, and A. Divakaran, "Automatic food documentation and volume computation using digital imaging and electronic transmission," Journal of the American Dietetic Association, vol. 110, no. 1, pp. 42-44, 2010.

[20] C. K. Martin, S. Kaya, and B. K. Gunturk, "Quantification of food intake using food image analysis," in Engineering in Medicine and Biology Society, 2009. EMBC 2009. Annual International Conference of the IEEE. IEEE, 2009, pp. 6869-6872.

[21] M. Puri, Z. Zhiwei, Y. Qian, A. Divakaran, and H. Sawhney, "Recognition and volume estimation of food intake using a mobile device," in Workshop on Applications of Computer Vision, 2009. 
[22] M. Sun, L. E. Burke, Z.-H. Mao, Y. Chen, H.-C. Chen, Y. Bai, Y. Li, C. Li, and W. Jia, "ebutton: a wearable computer for health monitoring and personal assistance," in Proceedings of the 51st annual design automation conference. ACM, 2014, pp. 1-6.

[23] A. Berenguer, J. Goncalves, S. Hosio, D. Ferreira, T. Anagnostopoulos, and V. Kostakos, "Are smartphones ubiquitous?: An in-depth survey of smartphone adoption by seniors," IEEE Consumer Electronics Magazine, vol. 6, no. 1, pp. 104-110, 2017.

[24] P.-Y. P. Chi, J.-H. Chen, H.-H. Chu, and J.-L. Lo, "Enabling calorieaware cooking in a smart kitchen," in International Conference on Persuasive Technology. Springer, 2008, pp. 116-127.

[25] K.-h. Chang, S.-y. Liu, H.-h. Chu, J. Y.-j. Hsu, C. Chen, T.-y. Lin, C.-y. Chen, and P. Huang, "The diet-aware dining table: Observing dietary behaviors over a tabletop surface," in International Conference on Pervasive Computing. Springer, 2006, pp. 366-382.

[26] R. S. Mattfeld, E. R. Muth, and A. Hoover, "Measuring the consumption of individual solid and liquid bites using a table embedded scale during unrestricted eating," IEEE journal of biomedical and health informatics, vol. 21, no. 6, pp. 1711-1718, 2017.

[27] B. Zhou, J. Cheng, M. Sundholm, A. Reiss, W. Huang, O. Amft, and P. Lukowicz, "Smart table surface: A novel approach to pervasive dining monitoring," in Pervasive Computing and Communications (PerCom), 2015 IEEE International Conference on. IEEE, 2015, pp. 155-162.

[28] G. Mertes, G. Christiaensen, H. Hallez, S. Verslype, W. Chen, and B. Vanrumste, "Measuring weight and location of individual bites using a sensor augmented smart plate," in Proc. of the 40th Annual International Conference of the IEEE Engineering in Medicine and Biology Society (EMBC). IEEE, 2018.

[29] V. Papapanagiotou, C. Diou, B. Langlet, I. Ioakimidis, and A. Delopoulos, "Automated extraction of food intake indicators from continuous meal weight measurements," in International Conference on Bioinformatics and Biomedical Engineering. Springer, 2015, pp. 35-46.

[30] V. Papapanagiotou, C. Diou, I. Ioakimidis, P. Sodersten, and A. Delopoulos, "Automatic analysis of food intake and meal microstructure based on continuous weight measurements," IEEE Journal of Biomedical and Health Informatics, 2018.

[31] L. Breiman, "Random Forrests," Machine learning, 2001.

[32] S. F. Simmons and D. Reuben, "Nutritional intake monitoring for nursing home residents: A comparison of staff documentation, direct observation, and photography methods," Journal of the American Geriatrics Society, vol. 48, no. 2, pp. 209-213, 22000. 